\title{
Meskhenet et les sept Hathors en Egypte ancienne
}

\section{Cathie Spieser}

\section{OpenEdition}

Journals

Édition électronique

URL : http://journals.openedition.org/edl/141

DOI : $10.4000 /$ edl. 141

ISSN : 2296-5084

\section{Éditeur}

Université de Lausanne

\section{Édition imprimée}

Date de publication : 15 décembre 2011

Pagination : 63-92

ISBN : 978-2-940331-26-0

ISSN : 0014-2026

\section{Référence électronique}

Cathie Spieser, « Meskhenet et les sept Hathors en Egypte ancienne », Études de lettres [En ligne], 3-4 I 2011, mis en ligne le 15 décembre 2014, consulté le 18 décembre 2020. URL : http:// journals.openedition.org/edl/141 ; DOI : https://doi.org/10.4000/edl.141 


\section{MESKHENET ET LES SEPT HATHORS EN ÉGYPTE ANCIENNE}

Dans la littérature égyptienne, le papyrus Westcar relate l'intervention déterminante des déesses Isis et Meskhenet pour le destin du nouveau-né. D’autres divinités, apparaissant dans différentes sources, jouent un rôle très important dans la naissance, la protection, la destinée et la durée de vie. A partir du Nouvel Empire et jusqu’à l'époque ptolémaïque, Hathor et Meskhenet se démultiplient pour former des collèges divins d'avatars qui, par leur nombre, accroissent leur puissance magique, mais aussi leur nature ambivalente et redoutable. Bien qu'elles n'en soient pas, elles agissent à la manière de "fées" veillant sur le nouveau-né. Comment s'articulent, dans les sources à disposition, les phases de la naissance de l'enfant royal? Quelles pratiques sociales et rites de passage se reflètent à travers ces sources?

\section{Des «contes de fées" égyptiens?}

Dans la littérature égyptienne, des textes considérés comme des "contes" relatent l'intervention de déesses annonçant le destin du nouveau-né ${ }^{1}$. Cette désignation relève cependant d'une catégorisation moderne qui cherche à rapprocher les textes anciens d'un genre connu, quand bien même il n'existe aucun terme qui corresponde au mot «conte» dans

I. Le terme «conte» est généralement employé pour désigner une catégorie bien spécifique de récits merveilleux de l'Egypte pharaonique. Le premier ouvrage paru sur les "contes» égyptiens est celui de G. Maspero, Les contes populaires de l'Egypte ancienne. Le terme n'a cessé d'être employé jusqu'à aujourd'hui, par exemple: C. Lalouette, Contes et récits de l'Egypte ancienne; E. Brunner-Traut, Altägyptische Märchen. 
la langue égyptienne, contrairement à d'autres genres littéraires bien documentés ${ }^{2}$.

G. Lefebvre, dans son ouvrage Romans et contes égyptiens de l'époque pharaonique publié en 1949, établit même une typologie des "contes", en distinguant les contes-cadres (qui renferment des prophéties ou des révélations), les contes mythologiques, les contes anecdotiques, les contes philosophiques, les contes psychologiques, ainsi qu'une catégorie plus large, celle des contes merveilleux, à laquelle appartiennent, selon lui, le Papyrus Westcar et le récit du «Prince prédestiné» qui vont tout particulièrement retenir notre attention. Ces compositions présentent aussi des éléments mythologiques, notamment des déesses qui prennent la parole et agissent, à l'instar d'autres personnages et même d'animaux fantastiques. Il est donc hasardeux de les réduire à des catégories spécifiques. Les "contes" égyptiens constituent, avant tout, des histoires ou des récits ressortissant, pour beaucoup d'entre eux, du merveilleux mêlant croyances, cultes et pratiques.

Certaines compositions, pour la plupart postérieures au Nouvel Empire, se présentent comme des «récits populaires» ${ }^{3}$. Il s'agit pourtant de véritables compositions littéraires recopiées maintes fois par des personnes qui ne pouvaient quappartenir à la minorité lettrée de la société égyptienne ${ }^{4}$. Cependant, à travers cette volonté de présenter ces œuvres comme "populaires", c'est surtout leurs destinataires que nous pouvons appréhender. Comme l'a relevé J.-L. Chappaz, la présence de particules communicatives dans les récits, placées souvent en début de paragraphe pour introduire un nouvel élément du récit, telles que $m . k, m$.tn que l'on traduit par "vois-tu", "voyez», laisse à penser que ces textes furent lus, ou contés, à un large public ${ }^{5}$. A cela s'ajoute l'adverbe $h r r$ que l'on traduit par "alors» qui sert souvent, lui aussi, à marquer le commencement d'un récit ou d'une partie importante dans un récit et signale ainsi

2. J.-L. Chappaz, "Quelques réflexions sur les conteurs dans la littérature égyptienne ancienne».

3. J. Baines, "Contexts of Fate». Voir aussi W. Helck, «Die Erzählung vom verwunschenen Prinzen».

4. J. Baines, "Contexts of Fate».

5. J.-L. Chappaz, "Quelques réflexions sur les conteurs dans la littérature égyptienne ancienne». 
le caractère narratif du texte: "Alors, il arriva ceci, cela, etc. ${ }^{6}$. Enfin, certains récits commencent par «Il était une fois» ou "Il y avait une fois», comme dans l'histoire du Paysan éloquent qui débute par "Il était (une fois) un homme»: $s p w w n$. Ainsi, ces histoires qui font appel au merveilleux et au surnaturel servaient sans doute au divertissement d'un large auditoire et rejoignent de ce fait la notion moderne de conte ${ }^{7}$.

Pour les Egyptiens anciens, la naissance est un événement que l'on doit protéger par des pratiques magico-religieuses parce qu'elle représente le moment décisif où se cristallisent la personnalité et le destin de l'individu. Les trois notions égyptiennes pour le sort, la chance (ou la fortune) et la destinée sont désignées par trois termes égyptiens différents: $\breve{s} 3 j j$, $r n n t$ et $m s h n t$. Elles sont également personnifiées par des divinités: le dieu Shay et son pendant féminin, la déesse Renenet, présents à la naissance, comme à la renaissance au moment de la pesée de l'âme, et Meskhenet, "le lieu de naissance» soit les briques de l'accouchement ${ }^{8}$. Ces divinités ont toutes en commun leur pouvoir sur la durée de vie de l'individu. Cependant, d'autres divinités pouvaient également intervenir en jetant des sorts qui pouvaient avoir une influence directe sur le destin d'un nouveau-né et sa durée de vie.

\section{Un accouchement en musique}

Dans le Papyrus Westcar, daté de l'époque Hyksos (vers 1600 av. J.-C.), mais dont le texte semble remonter à la fin du Moyen Empire, ce sont quatre déesses, Isis, Nephthys, Meskhenet et Héqet, qui interviennent pour assurer le bon déroulement de l'accouchement de la reine Redjejet et un destin favorable aux trois futurs pharaons de la $\mathrm{V}^{\mathrm{e}}$ dynastie. Pour passer "inaperçues» dans une forme terrestre, les quatre déesses vont d'abord se transformer en danseuses musiciennes. Avant d'entrer dans la chambre de la parturiente, elles agitent leurs sistres et leurs colliers

6. Cet adverbe se retrouve dans de nombreux récits: Sinouhé, le Naufragé, le Paysan éloquent, etc.

7. H. Simon, «Die Jungfrau im Turm»: le texte, par sa structure, son style et sa fonction de divertissement, justifie sa classification dans la catégorie des contes.

8. J. Baines, "Contexts of Fate»; Ch. Seeber, Untersuchungen zur Darstellung des Totengerichts, p. 83-88. 


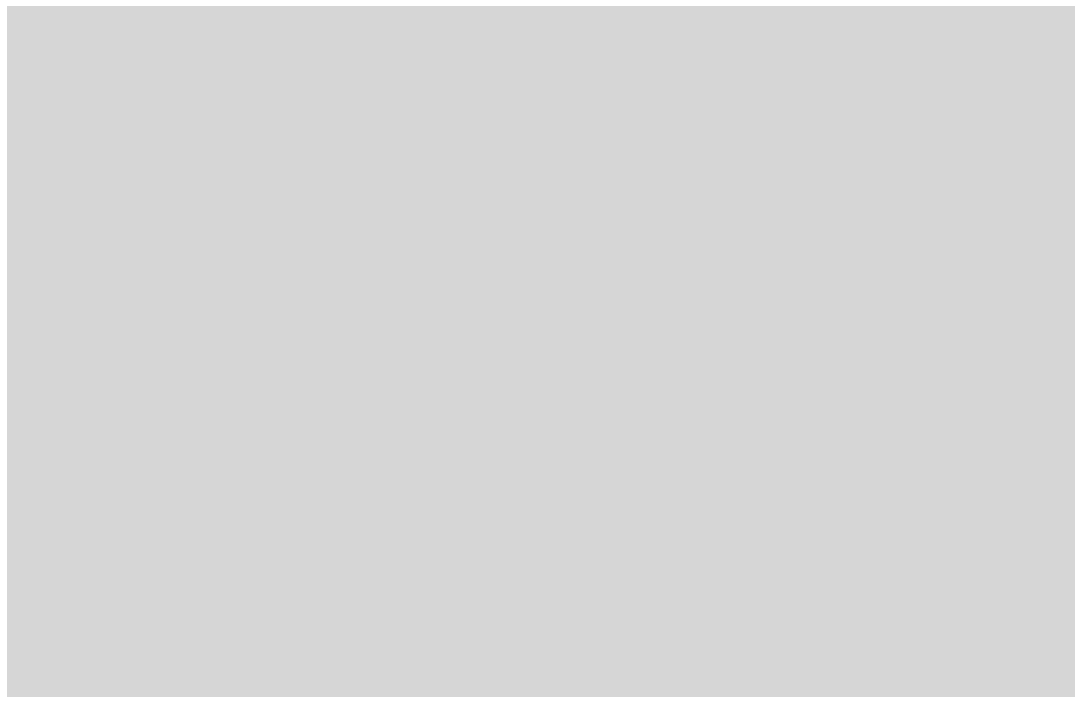

Fig. 1 - Ostracon, Deir el Médineh, Nouvel Empire.

ménat ${ }^{9}$. La musique, faite de cliquetis aigus, écarte les influences négatives et invisibles. Des divinités musiciennes et protectrices sont familières des scènes de naissance égyptiennes, figurées généralement au Nouvel Empire sur des ostracas (fragments de poterie) qui montrent l'accouchée sur un lit ou assise sur un siège tenant son enfant sous un kiosque orné de lierre (fig. 1) ${ }^{10}$. La musique et la danse prophylactique accompagnaient aussi les funérailles ${ }^{11}$. La musique marquait à la fois l'entrée dans la vie, ainsi que la disparition dans la mort qui devait elle-même conduire à une renaissance à une forme de vie éthérée. La musique accompagnait les accouchements des Egyptiennes et cette

9. Le collier ménat est composé d'un contrepoids métallique et de nombreuses rangées de perles en fritte émaillée qui, par leur frottement, donnent un son similaire à celui d'un sistre. E. Staehelin, «Menit».

Io. A. R. Schulman, "A Birth Scene from Memphis»; E. Brunner-Traut, Die Alten Aegypter, p. 56-60.

II. De l'Ancien Empire jusqu'au Nouvel Empire, c'est notamment le rôle des «danseurs Muu» de danser et de surveiller à la fois le transport de la momie vers son caveau. Voir, par exemple, la tombe thébaine de Reckmiré (Nouvel Empire) avec la représentation de danseurs Muu: S. Hodel-Hoenes, Leben und Tod im Alten Ägypten, Thebanische Privatgräber des Neuen Reiches, p. 130 et fig. 63. Ce sont aussi parfois des couples de jeunes filles ou de jeunes hommes figurés en train de danser aux funérailles: cf. E. Brunner-Traut, «Tanz». 
tradition, encore présente dans quelques sociétés africaines, notamment chez les pygmées Aka, se rattache peut-être à un ancien fond religieux africain. Enfin, cette musique bienfaisante et protectrice peut aussi être rapprochée de celle que les prêtresses étaient chargées de dispenser dans les temples, à proximité de la chapelle contenant la statue divine où l'esprit divin devait se réincarner quotidiennement ${ }^{12}$. Il est connu que les palais royaux et des maisons privées de hauts dignitaires étaient conçus selon un modèle d'agencement des pièces rappelant les temples. Dans la maison, la chambre à coucher correspondait au saint des saints, or c'est justement l'endroit où accouche Redjejet.

\section{Les déesses sages-fermmes: des "bonnes fées" accomplissant des prodiges}

Dans le papyrus Westcar, l'intervention des déesses Isis, Nephthys, Héqet et Meskhenet est clairement décrite ${ }^{13}$ : «Alors Isis se plaça devant elle (Redjedet), Nephthys derrière elle et Héqet accéléra la naissance.» Isis et Nephthys sont des déesses sœurs qui veillent sur Osiris, le prototype du roi défunt divinisé, et sur Horus, l'enfant héritier du trône royal. Héqet, la déesse à forme de grenouille, incarne et protège tout à la fois, le ventre et le mouvement des jambes repliées de la parturiente, rappelant le saut des grenouilles, qui devait faciliter la délivrance. Héqet est liée au principe de la fécondité de la vie, de la naissance et de la renaissance. Les déesses jouent manifestement le rôle de quatre sages-femmes. Elles déclarent d'ailleurs: "Nous savons faire un accouchement.» Ce passage qui décrit avec beaucoup de réalisme l'emplacement occupé par les sages-femmes autour de la parturiente qui devait être maintenue presque debout, et aidée pendant l'accouchement, reflète l'existence de sagesfemmes en Egypte ancienne, comme il en existait aussi dans d'autres civilisations.

Lorsque l'enfant glisse dans les mains de la déesse Isis, elle fait alors un jeu de mots qui forme le nom du premier enfant royal: Ouser-kaf, dont le nom signifie «Son esprit ka est puissant». La déesse fait de même

I2. S. Sauneron, Les Prêtres de l'ancienne Egypte, p. 78-83 et 94: la musique rythmait les offices religieux mais il existait aussi une "aubade musicale qui réveille le dieu au matin». Un hymne était chanté le matin pour «réveiller» le dieu.

13. G. Lefebvre, Romans et contes égyptiens de l'époque pharaonique, p. 86-90. 
pour les deux autres enfants. Le nom donné à l'enfant au moment de sa naissance constitue déjà une forme de prédiction, une caractéristique qui demeurera liée à l'individu. En fait, le mythe reflète ici une pratique sociale: le nom de l'enfant était en principe attribué par la mère et pouvait évoquer les premières paroles prononcées par la parturiente considérées comme un signe à caractère magique et protecteur pour l'enfant ${ }^{14}$.

L'étape suivante consiste à laver l'enfant et cette purification constitue un premier rite de passage: «Elles le lavèrent, après qu'eut été coupé son cordon ombilical et qu'il eut été placé sur un cadre de briques. " Ce cadre composé de deux briques, ou de quatre briques empilées par deux, placées de façon perpendiculaire et sur lesquelles la femme posait ses pieds, était probablement recouvert d'un rembourrage d'étoffe pour éviter de blesser l'enfant, comme l'indique un passage du papyrus Leiden I $3488^{15}$. La déesse Meskhenet annonce ensuite sa première prophétie: "un roi qui exercera la royauté dans ce pays entier». Ce n'est pas un hasard si l'intervention de la déesse se fait juste après le premier bain de l'enfant: il est propre et on peut faire un pronostic vital. Ce pronostic devient une prédiction. L'intervention du dieu Khnoum se fait après celle des déesses. Il n'assiste pas aux accouchements de Redjejet, mais il intervient juste après pour donner au nouveau-né la santé et la force. Khnoum n'est autre que la transposition de la figure paternelle qui marque un autre rite de passage: celui de la reconnaissance de l'enfant par le père.

I4. P. Vernus, «Namensgebung»; G. Posener, "Sur l'attribution du nom à un enfant». D'autres noms se rattachaient à des oracles, par exemple: «Le dieu X a dit qu'il vivra" et sont à considérer comme de véritables talismans pour leur porteur, cf. J. Sainte-Fare-Garnot, "Défis au destin"; P. Vernus, "Namensgebung", col. 328 et n. 25 à 28. D'autres noms indiquent que l'enfant est né grâce à l'intervention d'un dieu ou d'une déesse auquel avait été adressée une prière, par exemple: "c'est à Mout que j'ai demandé un rejeton», "le fils/la fille de la divinité X», etc. Cette dimension performative de la parole caractéristique des cultures antiques se retrouve dans la plupart des versions du conte de La Belle au bois dormant, comme le montre D. Haase dans sa contribution à ce volume.

15. J. F. Borghouts, The Magical Texts of the Papyrus Leiden I 348, cf. 4, recto 2.9., p. 16 sq., pl. 2 et 19: "une brique en tissu nd»; A. Gnirs, "Nilpferdstosszähne und Schlangenstäbe», p. 136, n. 62; E. Staehelin, «Bindung und Entbindung». A. Macy Roth, C. Roehrig, "Magical Bricks and the Bricks of Birth", p. 132, n. 62. Un texte magico-médical mentionne clairement l'utilisation de deux briques pour l'accouchement. L'emploi de deux briques devait être plus fréquent si l'on tient compte des parallèles ethnographiques. 
Les opérations sont identiques pour les deux autres enfants royaux: aide de Nephthys et Héqet, réception de l'enfant par Isis, prononciation du nom par Isis, coupure du cordon en même temps que l'enfant est placé sur les «briques», puis lavage du corps de l'enfant, et enfin prophétie de Meskhenet et intervention finale de Khnoum. Redjejet respecte ensuite une purification de quatorze jours. Les parturientes subissaient également une période de purification de quatorze jours durant lesquels elles demeuraient sans doute dans un kiosque construit spécialement pour l'occasion et qui devait se situer dans la cour de la maison ${ }^{16}$.

Ces étapes de la naissance des trois rois et le rite de purification de la reine du papyrus Westcar sont clairement la transposition de pratiques réelles. Le récit mentionne aussi un détail particulier: les déesses laissent un sac d'orge renfermant les couronnes des trois rois et dont s'échappe une musique de fête: "un bruit de chant, de musique, de danse, d'acclamations, tout ce qu'on a coutume de faire pour un roi $\%$. Ce fait intervient juste après qu'Isis et les déesses, une fois retournées chez elles, manifestent le désir de «faire pour ces enfants un prodige» qu'elles pourraient annoncer à leur père véritable, le dieu Rê. L'orge servait à faire de la bière. Nous avons ici l'évocation du retour des accouchées qui, au terme de leur purification, donnait lieu à une fête particulière en l'honneur de la naissance des enfants.

Isis, Meskhenet et d'autres dieux ou déesses du panthéon égyptien sont à même de faire des présages favorables à l'enfant royal. Les rôles des dieux et déesses peuvent se recouper entre eux, même si les attributions de chacun ne sont jamais tout à fait les mêmes et évoluent dans le temps.

Meskhenet joue cependant un rôle particulier dans la prédiction liée à la naissance. L'idée même de la naissance était personnifiée par la déesse Meskhenet dont le nom signifie «lieu d'accouchement", c'est-à-dire le siège de parturition composé de deux ou quatre briques qui permet à la femme d'accoucher en position accroupie. Meskhenet possédait un rôle parallèle dans le domaine funéraire. Ainsi, dans le chapitre de la pesée du cœur, elle figure, sous forme d'une brique à tête humaine, près de la balance qui indiquera si le mort peut accéder à la vie éternelle ou s'il sera jeté en pâture à la dévoreuse (fig. 2) ${ }^{17}$. Elle représente le destin du mort qui est momentanément mis en suspens.

I6. Je rejoins tout à fait l'avis d'A. Gnirs, «Nilpferdstosszähne und Schlangenstäbe».

17. Ch. Seeber, Untersuchungen zur Darstellung des Totengerichts im alten Aegypten, p. 83-84, fig. 24 et 25. 
Fig. 2 - Meskhenet sous forme de brique à tête humaine.

Cette déesse, très ancienne, était déjà mentionnée dans les Textes des Pyramides où son nom a pour signe déterminatif un utérus, probablement celui d'une vache $^{18}$. Ce signe en forme de crochet à double volutes sert aussi d'emblème à la déesse qui le porte volontiers sur la tête. Que cet élément ait été emprunté à une vache n'a rien d'étonnant: les Egyptiens associaient cet animal, considéré comme aquatique car vivant dans les marécages du Delta, à l'idée de beauté et de fécondité. La déesse Hathor fortement liée à l'univers de la naissance peut aussi être figurée sous les traits d'une vache ou avec des attributs de vache et elle possède un rôle comparable à celui de Meskhenet.

Dans une formule magique du papyrus Berlin 3027, datant du Nouvel Empire (1550-1070 av. J.-C.), destinée à «séparer l'enfant naissant du corps de sa mère", à prononcer sur les deux briques d'accouchement, Meskhenet est la déesse qui "procure l'esprit» à l'enfant qui est dans le ventre de sa mère ${ }^{19}$ :

[...] Il vient au monde l'enfant, tu sais (en ton nom) Meskhenet, comment procurer l'esprit à cet enfant qui est au flanc de cette femme. Tu lui procureras l'ordre royal donné à Geb, de créer l'esprit, l'âme et tout ce qu'il faut de Nout, et les maillots pour l'enfant de cette femme. Ne permets pas que soit prononcé aucun maléfice, car tu es bonne! Que ceux qui sont pris par la faiblesse, n'arrêtent pas ce qui est juste, avec de méchantes bouches! [...]. Cette formule doit être prononcée par le lecteur des saints livres sur deux briques sur lesquelles est assise la femme qui enfante.

I8. J. P. Allen, The Ancient Egyptian Pyramid Texts, p. 139: P 356; p. 159: P 464 et p. 326: Nt 244. Le signe déterminatif est le signe non phonétique qui indique le sens général du mot.

19. F. Lexa, La magie dans l'Egypte antique de l'Ancien Empire à l'époque copte, II, p. $29, \mathrm{~V}-5 / 8$ et $6 / 8$. 
Ainsi, la déesse insuffle non seulement "l'esprit", «l'âme» et "tout le nécessaire», mais elle procure aussi les maillots pour l'enfant. Ces bandes entourent son corps et lui offrent une protection magique. Meskhenet doit aussi le protéger contre les maléfices qui pourraient être prononcés contre lui. Ceci rappelle «le mauvais œil»si présent dans les pays orientaux: on se méfie d'un mauvais regard, ou de mauvaises paroles, en raison de la valeur créatrice attribuée aux mots écrits ou prononcés ${ }^{20}$.

Dans les temples ptolémaïques, Meskhenet apparaît sous forme d'un collège de quatre divinités (fig. 3) ${ }^{21}$. La démultiplication de la déesse a pour objectif de renforcer la puissance de son pouvoir magique et son ubiquité: le chiffre quatre représente généralement les directions cardinales et exprime une idée de totalité. Les quatre déesses possèdent des épithètes distinctes qui les assimilent, dans leur nature, à des déesses bien connues du panthéon égyptien. Ainsi, Meskhenet neferet (la belle) est identifiée à Isis, Meskhenet ouret (la grande/la puissante) à Tefnout, Meskhenet âat (la grande) à Nout et Meskhenet meneket (l'utile) à Nephthys ${ }^{22}$. Les quatre déesses assistent à la naissance d'Horus et sont des compagnes de Khnoum qui crée le corps de l'enfant sur son tour de potier $^{23}$. Les Meskhenet agissent ainsi à la manière de "bonnes fées" pour le divin nourrisson en lui servant de nourrice et en déterminant son héritage, ses héritiers, sa royauté et la durée de sa vie ${ }^{24}$. Elles lui accordent des bienfaits ou "dons»: courage, vie, santé, force. Au temple d'Esna, une inscription qui accompagne leur représentation indique:

20. Voir n. 14.

2I. Voir par exemple la belle illustration dans A. Mariette, Denderah, II, pl. 43b.

22. Elles sont appelées "Meskhenet neferet Isis", ainsi de suite.

23. Ce dernier pouvait aussi endosser le rôle de médecin accoucheur. Une inscription du mammisi de Philae indique: "Il est celui qui a fait accoucher sa sœur; celui qui a affermi la couronne blanche sur sa tête, dans ses domaines sur terre. Il l'a fait se lever sur les cuisses de sa mère, le jour de sa naissance... le jour de mettre au monde Horus", cf. F. Daumas, Les mammisis des temples égyptiens, p. 427-428.

24. Pour le rôle de Meskhenet à l'époque ptolémaïque: M. Derchain-Urtel, Synkretismus in ägyptischer Ikonographie, p. 29-34. 
Fig. 3 - Dendera, les quatre Meskhenet.

Ce sont elles qui comptent le temps, qui conjurent le mal, qui embellissent les lieux de naissance sur les quatre briques de naissance. Elles (fixent) son destin auprès du Maître du Tour de potier, pour créer ta famille dans $[\ldots]^{25}$

Un autre texte provenant du mammisi de Dendera montre le rôle fondamental des paroles de Meskhenet pour déterminer le destin du jeune Horus:

Paroles prononcées par Meskhenet la belle: je suis venue et je t'ai apporté toute la vie et le bien-être, toute la santé et toute la joie, tous les aliments et toutes les offrandes. Je rajeunis $(r n p j)$ [...] l'enfant, qui sort de ton corps. Je le place en tant qu'hériter du trône d'Horus et de tout ce que le soleil entoure ${ }^{26}$.

Le dieu solaire vieilli par sa course diurne est rajeuni par la déesse qui fixe aussi la durée de la grossesse. Une autre inscription prête à Meskhenet

25. S. Sauneron, Le temple d'Esna, p. 228-229, n³11, 17-19; M. Derchain-Urtel, Synkretismus in ägyptischer Ikonographie, p. 31, no 24.

26. Ibid., p. 32, no 29 . 
"l'utile» assimilée à Nephthys et, dans ce cas, aussi à Seshat, la déesse de l'écriture, un rôle tout aussi déterminant:

Paroles prononcées par Meskhenet l'Utile, Nephthys, la sœur du dieu, qui compte toute la durée de vie et toutes les années, le destin, la nourrice qui ordonne la venue à l'existence, qui fait des plans d'avenir pour Ounnefer ${ }^{27}$.

A travers ces divers exemples, Meskhenet semble prendre de l'importance au fil du temps. Son rôle, au moment de l'accouchement, s'est étendu à la durée de la grossesse et inclut le moment clé de la délivrance: c'est elle qui déclenche l'accouchement. Elle contribue, par les nombreux bienfaits octroyés au divin nouveau-né, à sa bonne destinée. Des découvertes archéologiques récentes de sièges de parturition, les fameuses «briques d'accouchement" comportant des représentations de divinités et probablement réservées à l'élite de la société égyptienne, tendent à montrer la continuité entre les données religieuses fournies par les temples et les croyances privées ${ }^{28}$.

\section{Hathor et les Sept Hathors dans leur fonction de "fée" pour le nouveau-né}

Hathor, une déesse figurée sous forme de vache ou purement anthropomorphe, ou avec une tête de vache sur un corps humain, était mise en relation avec l'amour, la joie, la musique, l'ivresse, la fécondité, la naissance et les prédictions à la naissance ${ }^{29}$. Toutes ces attributions sont aussi étroitement associées à l'accouchement. La déesse Hathor est appelée à Dendera "Maîtresse de Shaï et de Renenet ", c'est-à-dire maîtresse du destin et de son pendant féminin, qui signifie "la nourrice» ${ }^{30}$, Renenet

27. Ibid., p. 30, no 16; A. Mariette, Denderah, II, pl. 43b.

28. Comme par exemple le siège de parturition découvert à Abydos: J. Wegner, «A Decorated Birth-brick from South Abydos", p. 3 sq.

29. Sur Hathor, ses nombreuses variantes et épithètes: Ch. Leitz, Lexicon der Aegyptischen Götter und Götterbezeichnungen, V, p. 75-86.

30. Le nom de Renenet est basé sur la racine $r n n$ "nourrir, cultiver» et le déterminatif est celui d'une femme allaitant un enfant ou un serpent (Népri), cf. R. Hari, «La grande-en-magie de la stèle du temple de Ptah à Karnak», p. 101; F. T. Miosi, "God, Fate and Free Will in Egyptian Wisdom Literature», p. 75. 
étant la déesse de l'accomplissement, de la chance. Ces deux divinités, qui obéissent à Hathor, sont particulièrement associées aux présages de naissance et on les figurait parfois, à la fin du Nouvel Empire, comme des briques à tête humaine à la manière de Meskhenet ${ }^{31}$. Cette iconographie montre que, dans l'esprit des Egyptiens, le destin du nouveau-né se jouait sur les briques d'accouchement, sur le lieu de son premier examen physique qui permettait de poser un premier pronostic vital: l'enfant était-il complet, normal ou atteint d'une anomalie, d'une difformité? Semblait-il en bonne santé? Autant d'inquiétudes qu'il fallait écarter, en priorité, par un examen minutieux de son corps.

Comme les quatre Meskhenet, Hathor se multiplie pour former un collège puissant de sept déesses qui, à la manière des sept fées du conte de Perrault La Belle aux bois dormant, vont se pencher sur le nouveau-né pour délivrer leurs présages ${ }^{32}$. Le chiffre sept $(s f h)$ commun à de nombreux groupes d'entités divines, était sacré et peut-être lié à la notion de "délier», comme dans des expressions telles que "se défaire du mal», "séparer l'enfant de la mère" ${ }^{33}$. L'existence des Sept Hathors, attestée notamment par des récits datant du Nouvel Empire, est cependant plus ancienne que celle des quatre Meskhenet ${ }^{34}$. Les Sept Hathors étaient précédées par un groupe divin de sept vaches célestes mentionnées dans les chapitres 141 et 148 du Livre des Morts ${ }^{35}$. Ces vaches portent toutes la ménat ${ }^{36}$, un attribut d'Hathor, mais seules trois d'entre elles ont des noms qui les rapprochent de la déesse, tandis que les autres évoquent

3I. F. T. Miosi, "God, Fate and Free Will in Egyptian Wisdom Literature»; Ch. Seeber, Untersuchungen zur Darstellung des Totengerichts im Alten Aegypten, p. 85.

32. Le fait qu'elles n'aient pas de nom individuel comme les Meskhenet, mais uniquement des épithètes, ne diminue pas leur importance; elles ne sont pas non plus des «fées», contrairement à ce qu’ffirme P. Hubaï, «Eine litterarishe Quelle der ägyptischer Religionsphilosophie», p. 280.

33. A. Erman, H. Grapow, Wörterbuch der Aegyptischen Sprache, IV, 115.

34. Les premières mentions des Meskhenet (au pluriel) comme entités formant un collège divin de nombre indéterminé datent du Nouvel Empire. Cependant les quatre Meskhenet apparaissent à l'époque gréco-romaine: cf. Ch. Leitz, Lexicon der Aegyptischen Götter und Götterbezeichnungen, p. 440-441.

35. R. El-Sayed, «Les sept vaches célestes, leur taureau et les quatre gouvernails». Une première mention des sept vaches se trouve sur une stèle abydénienne datant $\mathrm{du}$ Moyen Empire. L'inscription, qui constitue un prototype du chapitre 148 du Livre des Morts, donne les noms des sept vaches.

36. A propos de la ménat, voir n. 7. 
leur fonction ou un lieu particulier de l'univers funéraire. Ces vaches divines étaient invoquées pour assurer au mort la nourriture nécessaire à son existence d'outre-tombe. Mais elles n'étaient pas uniquement des nourrices pour le mort ${ }^{37}$; elles étaient aussi chargées d'assurer sa renaissance ${ }^{38}$. Les sept vaches célestes sont accompagnées d'un taureau et de quatre gouvernails qui sont aussi des entités protectrices et nourricières. L'une des vaches, appelée Khenemet ankh, «elle préserve la vie» ou "nourrice de vie», devait tout particulièrement permettre au mort de renaître à la vie. Elle est parfois figurée avec un pelage rouge. Cette vache est aussi désignée par le terme jnwtt, "la colorée " ${ }^{39}$, et parfois aussi jns, avec déterminatif de l'étoffe, «la bandelette rouge " ${ }^{40}$. Cette bandelette de couleur rouge clair doit rappeler le sang, en vertu d'un symbolisme que nous allons aussi retrouver chez Hathor. Les sept vaches célestes continuent à exister, parallèlement aux Sept Hathors, jusqu'à l'époque ptolémaïque ${ }^{41}$, où leurs noms individuels changent, et où elles constituent désormais un groupe divin plus étroitement lié à Hathor, celui des «Sept vaches de la Dorée». Ces vaches, qui ont probablement contribué à l'émergence des Sept Hathors, resteront un collège divin distinct de celui des Sept Hathors qui sont figurées de manière anthropomorphe.

On trouve les Sept Hathors dans deux histoires égyptiennes datant du Nouvel Empire. Le "Prince prédestiné», du pap. Harris 500, datant des XIX ${ }^{\mathrm{e}}-\mathrm{XX}$ dynasties (ramesside), raconte qu'un roi avait imploré les dieux pour obtenir un fils ${ }^{42}$. Après sa naissance, les Sept Hathors sont venues pour lui fixer son destin (shai) en prononçant des présages (shaw). Elles

37. Elles sont aussi les nourrices de Rê. Voir R. El Sayed, «Les sept vaches célestes, leur taureau et les quatre gouvernails", p. 375-376.

38. Les formules doivent permettre au mort de "venir à l'existence sous vos croupes", cf. R. El Sayed, «Les sept vaches célestes, leur taureau et les quatre gouvernails», p. 359 et 373 .

39. Par exemple, dans la tombe de Nefertari: H. Schmidt, J. Willeitner, Nefertari Gemahlin Ramses'II, p. 139, fig. 200.

40. R. El-Sayed, "Les sept vaches célestes, leur taureau et les quatre gouvernails", p. 363-369. Voir aussi l'article de V. Dasen dans ce volume.

4I. Elles sont figurées à Edfou, Kom Ombo et Denderah: cf. R. El-Sayed, «Les sept vaches célestes, leur taureau et les quatre gouvernails», p. 385. Pour Dendera, cf. E. Chassinat, F. Daumas, Le temple de Dendera, VII, p. 124 et pl. DCXLIII.

42. G. Lefebvre, Romans et contes égyptiens de l'époque pharaonique, p. 114-124; W. Helck, "Die Erzählung vom verwunschenen Prinzen». Ce récit est à caractère politique. Voir ausi, H. Simon «Die Jungfrau im Turm». 
Fig. 4 - Dendera, crypte sud nº 2 , les Sept Hathors précédées de la déesse Ouadjet. 
prédisent un sort funeste: «il périra par le crocodile ou par le serpent, ou encore par le chien». Malgré ces prédictions, le récit, dont il manque la fin, finit probablement bien. Ces mêmes déesses énoncent aussi un destin fâcheux dans l'histoire «des Deux Frères" datant de la fin de la XIX dynastie, époque Mineptah-Siptah (pap. d'Orbiney, BM 10183, 9, 8-9) ${ }^{43}$. Les Sept Hathors rendent visite à la femme que Khnoum a créée pour le magicien Bata et annoncent que celle-ci "mourra par le glaive» en raison de son infidélité. Les déesses incarnent à la fois le destin et le retournement du destin contre ceux qui commettent des actes injustes. Un héros peut aussi, par ses actions, déjouer les mauvais présages. Le rôle des Sept Hathors consiste autant à fixer la durée de vie que le moment de la mort, ainsi que les circonstances qui les entourent ${ }^{44}$. L'histoire «des Deux Frères» connaît, elle aussi, une issue heureuse.

Dans les temples et les mammisis (temples dédiés à la naissance d'Horus et de ses avatars) gréco-romains, les Sept Hathors jouent un rôle plus pacifique et même pacificateur (fig. 4$)^{45}$. D'un temple à l'autre, ce ne sont jamais exactement les mêmes déesses, dont les épithètes peuvent varier, qui sont figurées ${ }^{46}$. Leurs titres peuvent évoquer leurs qualités, leur rôle et une partie d'entre eux sont des épithètes géographiques construites sur le modèle: "Hathor, maîtresse du (lieu)». Au temple d'Hathor à Dendera, des reliefs de l'ouâbet montrent les sept déesses qui dansent et jouent du tambourin pour accueillir et réjouir la déesse Hathor appelée sous différents noms et épithètes: la Dorée, la Régente, la Maîtresse des Deux Terres, l'CEil de Rê, la Vénérable et Puissante, etc. ${ }^{47}$. Ces épithètes rappellent la double nature de la déesse Hathor, dotée d'une puissance redoutable lorsqu'elle revient du sud, incarnant

43. Le contenu de ce récit possède une valeur initiatique dans la mesure où le héros meurt et renaît sous une forme différente; il est ensuite «identifié» et «réintégré», c'està-dire que le héros est reconnu dans sa nouvelle apparence, puis retrouve la place qui lui revient. J. Assmann, «Das Aegyptische Zweibrüder Märchen».

44. S. Morenz, Untersuchungen zur Rolle des Schicksals in der ägyptischen Religion, p. 32-34.

45. F. Daumas, "Sur deux chants liturgiques des mammisis de Dendera»: un hymne présent à la fois au mammisi de Nectanébo et au mammisi romain de Dendera scande l'apaisement de la déesse Hathor: Htp.n.s «apaisez-la» suivi du nom de différents dieux/ déesses.

46. F. Daumas, Les mammisis des temples égyptiens, p. 415-416, note 3; voir aussi M. Rochholz, Schöpfung, Feindvernichtung und Regeneration, p. 75-90.

47. S. Cauville, Dendera, IV, p. 30, 356-359, pl. XXV. 
le feu solaire qui brûle la terre et le désir de l'accouplement. Lorsque l'inondation, qui marque le Nouvel An, fait son œuvre, la déesse se pacifie à l'approche de sa prochaine maternité. Les eaux rougies de l'inondation évoquent un aspect ambivalent de son pouvoir: l'eau rouge représente le sang que la déesse réclamait dans le mythe de la vache du Ciel. Elle est aussi la bière rouge offerte à Hathor comme substitut du sang ${ }^{48}$. Hathor est alors appelée la Maîtresse de l'ivresse. C'est dans une ambiance festive faite de vin, de bière, de musique et de danse, que les Sept Hathors l'accueillent et lui procurent l'apaisement. Des chants liturgiques montrent que la musique des Hathors servait à attirer le divin époux Amon dans les mammisis de Dendera, pour son union avec elle, ainsi que pour la reconnaissance par le dieu du fils de la déesse ${ }^{49}$. L'ivresse, la musique et la danse sont les signes annonciateurs d'une issue heureuse: la naissance du jeune dieu solaire. Parmi les offrandes faites à la déesse Hathor se trouve notamment l' «étoffe rouge de Tayt " ${ }^{50}$. Tayt, dont le nom signifie "étoffe", est la très ancienne déesse du tissage déjà mentionnée dans les Textes des Pyramides: elle est celle qui "nouait» le corps du roi défunt, en lui "attachant ses membres" afin de lui assurer l'intégrité physique et la résurrection ${ }^{51}$. Ce faisant, elle remplissait aussi une fonction maternelle, en assurant la renaissance du roi mort ${ }^{52}$. Dans le rituel de l'embaumement, Tayt tisse les bandelettes qui servent à momifier le roi défunt ${ }^{53}$. Tayt est aussi le nom de l'étoffe rouge et ce n'est pas un hasard si cette étoffe est "celle qui s'ajuste sur le corps" ${ }^{54}$.

48. M.-Ch. Poo, Wine and Wine Offering in the Religion of Ancient Egypt, p. 155-158.

49. F. Daumas, «Sur deux chants liturgiques des mammisis de Dendera». Voir aussi l'évocation de l'accueil de la déesse Hathor en musique au temple de Philae, F. Daumas, "Les propylées du temple d'Hathor à Philae».

50. S. Cauville, Dendera, IV, p. 31-34.

51. J. P. Allen, The Ancient Egyptian Pyramid Texts, p. 22 : W 54 et p. 85: T 220-221.

52. Au Nouvel Empire, Tayet a été assimilée à Isis sous la forme d'Isis-Tayet. Tayet partage le rôle du tissage des bandelettes avec la déesse Neith de Sais. cf. H. El Saady, "Reflections on the Goddess Tayet».

53. J.-C. Goyon, Rituels funéraires de l'Ancienne Egypte, p. 146 et 155. Dans le conte de Sinouhé, Tayt pourvoit le héros de vêtements qu'elle a tissés de ses mains, cf. M. Lichtheim, Ancient Egyptian Literature, I, p. 229. Ce rôle de déesse du tissage est aussi partagé avec la déesse Neith comme l'indique le Texte des Sarcophages, sp. 608, cf. R. El-Sayed, «Les rôles attribués à la déesse Neith dans certains Textes des Cercueils", p. 286-293.

54. S. Cauville, Dendera, IV, p. 34. Un rapprochement pourrait être fait entre Tayt et Tabithet: D. T. M. Frankfurter, "Tabitha and the Apocalypse of Elijah». 
Elle joue un rôle identique aux bandelettes données par Meskhenet au dieu nouveau-né et peut probablement être rapprochée d'une des sept vaches célestes ${ }^{55}$. Dans un texte magique provenant d'un ostracon de Deir-el Médineh, les Sept Hathors sont également décrites comme porteuses de bandeaux rouges: "Salut à vous, vous les Sept Hathors qui êtes ornées de bandeaux de lin rouges... ${ }^{56}$. Ce bandeau rouge évoque aussi la création même de l'enfant dans le corps de la mère. La couleur rouge est associée au soleil naissant dans l'horizon céleste et elle évoque aussi le sang qui entoure la naissance, l'accouchement ${ }^{57}$. Le bandeau en luimême doit représenter le fil de la vie qui se crée, qui se tisse et probablement aussi le cordon ombilical, le lien qui unit la mère à l'enfant. Une formule magique destinée à prévenir et à guérir des piqûres de scorpion évoque les sept Hathors qui font sept nœuds avec leurs sept bandelettes:

[...] Les sept filles de Rê se lamentaient; elles firent sept nœuds de leurs sept bandeaux et sauvèrent celui qui avait été mordu (par un scorpion). Puisse-t-il se redresser, guéri pour sa mère, comme Horus s'est dressé, guéri pour sa mère Isis dans la nuit où il fut mordu! ${ }^{58}$

Ces nœuds sont protecteurs. De nombreuses formules magiques de recettes destinées à la protection de la femme enceinte ou de l'enfant mentionnent le recours à une amulette constituée d'un cordon que l'on doit nouer de sept nœuds ${ }^{59}$. Mais les sept déesses étaient aussi invoquées pour la protection du corps en général:

55. Cf. infra.

56. S. Schott, Altägyptische Liebeslieder, p. 85; J. F. Borghouts, Ancient Egyptian Magical Texts, p. 1, no 1 : ostracon Deir el-Médineh 1057; S. Morenz, Untersuchungen zur Rolle des Schicksals in der ägyptischen Religion, p. 34.

57. C. Spieser, "Le sang et la vie éternelle dans le culte solaire amarnien".

58. W. Pleyte, F. Rossi, Papyrus de Turin, pl. 135 et p. 12-13; J. F. Borghouts, Ancient Egyptian Magical Texts, p. 78, $\mathrm{n}^{\circ}$ 108, ostracon Deir el-Médineh 1048. Les «sept filles de Rê" sont probablement une autre désignation pour les Sept Hathors qui sont aussi associées aux sept scorpions qu'Isis créa pour la protection d'Horus, d'après la stèle Metternich (II. 51-62): M. Rochholtz, Schöpfung, Feindvernichtung und Regeneration, p. 215.

59. N. Yamazaki, Zaubersprüche für Mutter und Kind, par exemple p. 30, texte L, pour vaincre la maladie $s s m j$ : après la formule à réciter, "l'enfant ou sa mère doit manger une souris cuite. Ses os doivent être attachés à un cordon de lin fin à son cou, où l'on aura fait sept nœuds». Un autre exemple destiné à protéger le nouveau-né de l'esprit malfaisant d'un mort: «[...] On doit prononcer ce texte sur un sceau et dire d'une main: ce sera fait comme amulette, noué avec sept nœuds - avec un nœud le matin, un autre le soir, jusqu'à ce que l'on ait sept nœuds", cf. p. 42 sp. Q. D'autres exemples avec 
[...] Les Sept Hathors: elles vont veiller à la protection du corps jusqu’à ce que le corps soit sain [et ...] comme l'apparition de Rê sur le pays ${ }^{60}$.

A Dendera se trouvent deux reliefs consacrés aux Hathors qui sont liés à l'intronisation de la déesse ${ }^{61}$. Dans l'un, elles jouent du tambourin ${ }^{62}$; dans l'autre, ce sont trois Hathors qui frappent du tambourin et quatre qui jouent à l'aide de sistres de types différents: un sistre naos et un sistre hathorique ${ }^{63}$. Les sept déesses, en plus de leurs attributs hathoriques, portent la coiffe à vautour typique des déesses-mères. Hathor y reçoit diverses épithètes, parmi lesquelles: "la maîtresse du sistre naos", "la maîtresse du sistre crécelle». La déesse est aussi appelée "Soleil féminin»: «Nous jouons du sistre pour le Soleil féminin.» Le sistre est l'instrument qui devait favoriser l'épiphanie de la déesse. Dans ce même temple de Dendera, pas moins de douze Hathors musiciennes, associées à différentes localités, jouant du sistre et du tambourin, sont encore figurées dans la décoration entourant une niche (fig. 5) ${ }^{64}$. Celle-ci servait de réceptacle pour les statues divines de la déesse Hathor ${ }^{65}$. Le décor de la bordure inférieure de la niche montre que celle-ci est à interpréter comme étant "soulevée" par quatre déesses célestes soulevant le signe du ciel. Ainsi, la niche, destinée à contenir des effigies divines d'Hathor, constitue un espace céleste propice à l'apparition d'Hathor. Le rôle des Hathors musiciennes est clair: elles dansent et font de la musique pour favoriser sa présence divine dans la statue qui doit lui servir d'habitacle. Les épithètes de la déesse, la «Dorée», «celle qui brille comme l'or», la «Maîtresse du

utilisation de corde/bande avec un nombre de nœuds variable, à nouer autour du cou de l'enfant: p. 32, sp. M; p. 34 sp. N; p. 36 sp. O; p. 48-49, sp. U; p. 32, sp. V (sept nœuds).

6o. J. F. Borghouts, Ancient Egyptian Magical Texts, p. 46, nº 74, pap. Berlin 3038, (190), 21, 3-9.

6I. S. Cauville, Dendera, V-VI, p. 51, pl. LVI.

62. Ibid., p. 280-283 et pl. XXXXVI: crypte sud no 2, A, passages A-B; A. Mariette, Denderah, III, pl. 59.

63. S. Cauville, Dendera, V-VI, pl. LVI, p. 410; A. Mariette, Denderah, III, pl. 76.

64. E. Chassinat, Le temple de Dendera, III, pl. CLXXVI.

65. L'intérieur de la niche montre des représentations des formes animales d'Hathor, d'Isis-Ounyt, d'Horus et d'Harsomtous-Rê, les statues d'Hathor et d'Isis-Hathor et deux sistres sacrés, selon S. Cauville, Dendera, III, p. 5. Voir aussi S. Cauville, «Les statues cultuelles de Dendéra d'après les inscriptions pariétales». 
Fig. 5 - Dendera, niche entourée par douze Hathors musiciennes. 
Fig. 6 - Mammisi romain de Dendera. Hathors allaitant le dieu-enfant Ihy face à Khnoum créant l'enfant sur son tour de potier, et au roi offrant deux vases à lait.

ciel» montrent qu'Hathor est vénérée en sa qualité d'entité solaire ${ }^{66}$. Elle est le «disque solaire féminin égal du disque masculin». Bien qu'aucune déesse ne joue de la harpe, une inscription du côté droit du relief indique qu'on lui joue de la harpe ${ }^{67}$. Hathor est identifiée à "Meskhenet qui élève son Horus». Elle est aussi "Celle qui est pleine de vie, qui aime la bandelette rouge " ${ }^{68}$, soit une allusion au lien protecteur unissant la mère à l'enfant, probablement le cordon ombilical. Ce dernier trouve aussi son expression dans une amulette en forme de lien rouge, destinée à la protection de la naissance et la renaissance. Ce cordon rouge est lié notamment aux sept vaches du Livre des Morts, aux Sept Hathors, à Hathor, mais aussi au dieu chacal Anubis, portant un tel cordon rouge à son cou et qui joue un rôle important dans la renaissance des morts ${ }^{69}$.

La naissance et la renaissance sont également signifiées par l'allaitement. Au mammisi d'Edfou, un temple destiné à la célébration de la naissance du jeune dieu solaire, les Sept Hathors sont figurées en présence

66. S. Cauville, Dendera, III, p. 81-85.

67. Ibid., p. 85.

68. Ibid., p. 85.

69. Article en préparation sur «L'ambivalence du lait et des figues en Egypte ancienne", à paraître dans Mélanges dédiés à Françoise Dunand. 
d'Hathor allaitant un avatar du jeune dieu solaire, Harsomtous ${ }^{70}$. Au mammisi romain de Dendera, elles allaitent l'enfant face au dieu Khnoum figuré en train de créer l'enfant sur son tour, tandis que le roi fait une offrande de lait (fig. 6) ${ }^{71}$. Les déesses font partie du cycle narratif de la naissance du dieu solaire. Dans un autre registre, les mêmes déesses allaitent l'enfant de leur lait divin, gage de vie éternelle, tout en lui donnant différents bienfaits ${ }^{72}$. Ces dons sont autant de présages heureux destinés au jeune dieu. Au mammisi d'Edfou, au niveau du soubassement, c'est un collège de vingt déesses figurées de façon identique, parmi lesquelles nous retrouvons les Sept Hathors ( $\mathrm{n}^{\text {os }} 11$ à 17 ) qui donnent différents bienfaits destinés à assurer un bel avenir au dieu nouveau-né: «toute l'acclamation $(h n w)$, toute la joie, toute la santé, toute la vie, etc. ${ }^{73}$.

Au mammisi d'Edfou, l'accouchement de la déesse est conçu comme une mort rituelle (fig. 7). Pour permettre à la déesse de retrouver les forces qu'elle a perdues, le roi lui fait une offrande sous forme d'une galette et d'un pot de miel:

Je t'offre la galette $\check{s} s r$ jointe au miel pour remettre en état ton ventre après l'accouchement; tu manges les pains que tu as faits de tes mains, apaisée par les humeurs de ta majesté ${ }^{74}$.

Ces pains sont aussi appelés ta-mesout, les pains de naissance. Les travaux d'Irini Papaikonomou ont permis de faire la lumière sur l'existence de "pains de naissance», en Grèce ancienne, qui étaient assimilés au

70. E. Chassinat, Le mammisi d'Edfou, pl. XV, p. 32.

7I. F. Daumas, Les mammisis de Dendera, pl. XLVIII.

72. E. Chassinat, Le mammisi d'Edfou, p. 29-30. Même chose pour le mammisi romain de Dendera, cf. F. Daumas, Les mammisis des temples égyptiens, p. 415-418. L'enfant solaire reçoit des Sept Hathors du lait divin «blanc» et "lumineux».

73. E. Chassinat, Le mammisi d'Edfou, p. 11. Les Sept Hathors interviennent aussi au temple d'Edfou, dans les rites du second jour épagomène qui correspond à la naissance d'Horus: M. Alliot, Le culte d'Horus à Edfou au temps des Ptolémées, p. 369. Au mammisi romain de Dendera, les Sept Hathors sont également figurées au niveau du soubassement, jouant du sistre et du tambourin pour apaiser la déesse et protéger l'enfant divin, cf. F. Daumas, "Sur deux chants liturgiques des mammisis de Dendara», p. 44.

74. E. Chassinat, "A propos de deux tableaux du mammisi d'Edfou». Les "humeurs de ta majesté» sont une autre manière de désigner les offrandes faites par le roi, à savoir la galette et le miel. Les offrandes sont assimilées à des fluides précieux émanant du roi lui-même. Le texte fait aussi allusion à une pratique égyptienne: l'accouchée, après la délivrance, fabriquait une galette de ses propres mains et mangeait du miel afin d'apaiser ses douleurs et de soigner son ventre. 
placenta, appelé le gâteau de la mère, en allemand Mutterküchen: l'ingestion d'un tel gâteau devait permettre une "restitution" de ce dernier à l'accouchée ${ }^{75}$. Or le repas de la veille du Nouvel An, qui correspond au retour de l'inondation, était lui aussi composé de pain, le mesout tep renepet: "pain de Nouvel An». Une autre inscription d'Edfou indique que le roi offrait aussi à Hathor le "pain wp-r3», le pain de l'ouverture de la bouche, en référence à un rituel destiné à réveiller les morts à la vie ${ }^{76}$. L'accouchement était comparé à une mort de la déesse qui devait retrouver, à travers ces pains évoquant le placenta, la force vitale qu'elle avait perdue. Après son accouchement, la déesse Hathor subissait, elle aussi, une période de purification dans la hout ouâb, "le temple de la purification" ${ }^{77}$.

Des monuments privés attestent que les Sept Hathors étaient invoquées de manière personnelle pour obtenir notamment la naissance d'enfants, comme l'atteste une stèle-naos d'époque ramesside montrant le grand prêtre de Thot Amenemhat, au crâne rasé, les mains levées en signe de prière devant une niche dans laquelle se trouvent figurées en ronde-bosse les Sept Hathors ${ }^{78}$. Le texte dit ainsi :

Offrande que donne le roi, O vous les Sept Hathors! Puissent-elles donner ce qui provient d'elles, des liquides, des bœufs, des volailles, de l'eau fraîche, du vin, de l'encens, toutes choses bonnes et pures, desquelles vit un dieu. Puissent-elles donner des enfants des deux sexes, (ne pas) rester devant elles sans prononcer une offrande nombreuse par les personnes qui sont en présence (de moi) chaque jour. Je sors (de mon lieu?) pour la nourriture et des aliments et faire le bien, pour le $k a$ du favori de son Maître... ${ }^{79}$

La partie manquante de la stèle a été retrouvée; elle comportait une représentation de l'épouse du grand prêtre, la chanteuse d'Amon, Tamerout ${ }^{80}$. Enfin, un godet à onguent conservé au Musée du Louvre (E 25298)

75. I. Papaikonomou, S. Huysecom-Haxhi, «Du placenta aux figues sèches».

76. E. Chassinat, "A propos de deux tableaux du mammisi d'Edfou», p. 183-184.

77. Elle devait "purifier ses membres après l'accouchement", c'est-à-dire qu'elle recevait une purification à l'eau et des fumigations.

78. F. W. F. von Bissing, H. P. Block, «Eine Weihung an die sieben Hathoren»; M. Rochholz, Schöpfung, Feindvernichtung und Regeneration, p. 64, doc. 21.

79. "Ne pas rester devant elles sans prononcer une offrande» signifie que les personnes qui passent devant cette stèle sont exhortées à faire des offrandes.

80. D. Raue, "Die Sieben Hathoren von Prt». 


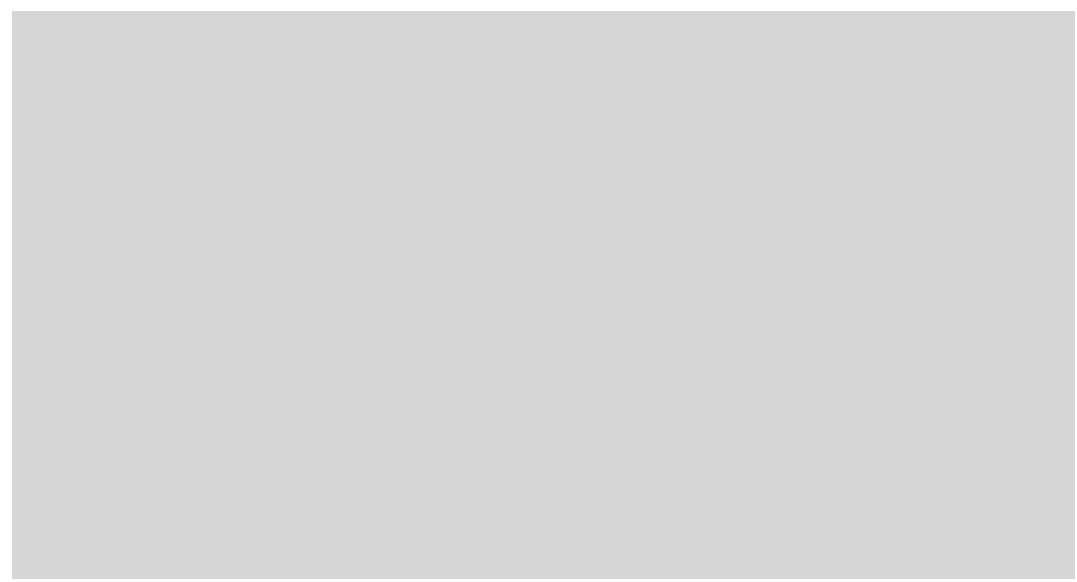

Fig. 7 - Mammisi d'Edfou. Hathor recevant une galette de naissance et du miel de la part du roi.

montre sur quatre faces des scènes en rapport avec la renaissance d'un mort ${ }^{81}$. Les Sept Hathors sont figurées en présence d'une défunte portant un cône funéraire et qui joue du sistre aux déesses; une autre face de l'objet montre l'égide d'Hathor, sa tête (disparue) encadrée d'un collier, ainsi que deux génies du Nil et un poisson inet, c'est-à-dire un tilapia nilotica qui pouvait représenter le corps du mort dans sa phase de régénération/momification ${ }^{82}$. Les animaux du désert figurés dans une marche pacifique représentent les forces hostiles maîtrisées, tandis que la scène de pêche au filet délivre un message identique: la défunte échappe à tous les dangers de l'au-delà. Ces scènes étaient destinées à favoriser la renaissance de la défunte placée sous la protection des Sept Hathors.

\section{Conclusion}

Pour conclure, différentes déesses, parmi lesquelles Isis, Meskhenet et Hathor, jouent un rôle important en tant que «bonnes fées» à la naissance

8I. Ch. Desroches-Noblecourt, «Un lac de turquoise, godets à onguents et destinées d'outre-tombe dans l'Egypte ancienne». M. Rochholz, Schöpfung, Feindvernichtung und Regeneration, p. 70 , doc. 34 .

82. E. Hornung, Geist der Pharaonenzeit, p. 181-200. En outre le mot «corps» s'écrivait avec le signe déterminatif du poisson. 
des enfants divins et royaux, un rôle qui demeure toutefois difficile à évaluer dans les croyances privées. Dans les sources écrites et monumentales, les grandes étapes de la naissance et certains événements comme le placement de l'enfant sur les briques qui correspond à l'examen de viabilité de l'enfant ou encore le don du nom à l'enfant, sont révélatrices de pratiques sociales et religieuses. Les déesses Hathor et Meskhenet se multiplient en collèges divins afin d'accroître leur puissance magique. Ce sont ces déesses qui fixent le destin du nouveau-né, mais aussi ses limites et même les conditions de sa mort. Dans les récits égyptiens, les présages néfastes ne sont pas fatals pour autant, mais ils peuvent être déjoués. Dans les temples gréco-romains, les Sept Hathors vont accueillir par leurs chants et leurs danses la déesse Hathor qui était partie en Nubie. Hathor, identifiée à Sekhmet, incarne la sécheresse mortelle précédant l'inondation. Son retour en Egypte, qui marque le Nouvel An, est célébré par les chants et les danses des Sept Hathors. L'apaisement ainsi procuré à la déesse annonce son changement de statut par sa prochaine maternité. Les quatre Meskhenet, tout comme les Sept Hathors sont à nouveau présentes au moment de l'accouchement de la déesse qui se fait en musique et vont procurer leurs bienfaits à l'enfant solaire. Le pouvoir des Sept Hathors est particulièrement mis en relation avec un bandeau rouge qui représente le lien unissant la mère à l'enfant, probablement le cordon ombilical, mais aussi le destin dont l'élaboration est comparée à un tissage. La déesse Tayt incarne l'étoffe rouge qui est à la fois un mode de renaissance et un linceul. L'usage de liens rouges à sept nœuds dans de nombreuses recettes médico-magiques destinées à la protection de la mère et de l'enfant fait référence à ces données religieuses. Nous retrouvons ce même bandeau rouge comme attribut des Sept vaches du Livre des Morts, d'Hathor et du chacal Anubis. Enfin, les Sept Hathors transmettaient aussi des dons et des bienfaits au nourrisson solaire par l'allaitement; le lait était, en effet, considéré comme un breuvage divin lié à la renaissance et à la vie éternelle. Une dernière étape liée à l'accouchement d'Hathor, figurée notamment au mammisi d'Edfou, consistait à lui offrir un "pain de naissance» afin de lui restituer, par ce biais, le placenta et de lui permettre de retrouver son intégrité et sa vitalité. Ce rite trouvait probablement aussi un écho dans les pratiques religieuses privées.

Cathie SpIEser Université de Fribourg 


\section{BIBLIOGRAPHIE}

Allen, James P., The Ancient Egyptian Pyramid Texts, Atlanta, Society of Biblical Literature, 2005.

Alliot, Maurice, Le culte d'Horus à Edfou au temps des Ptolémées, IFAO, Le Caire, 1954 (Bibliothèque d'Etudes XX).

Assmann, Jan, «Das Aegyptische Zweibrüder Märchen», Zeitschrift für Aegyptische Sprache, 104 (1977), p. 1-25.

Baines, John, "Contexts of Fate: Litterature and Practical Religion", in The Unbroken Reed. Studies in Honor of A. F. Shore, ed. by C. Eyre, A. Leahy, L. Montagno-Leahy, London, 1994, p. 35-52.

Bissing, Friedrich Wilhelm von, BLOck, Hans Peter, "Eine Weihung an die sieben Hathoren", Zeitschrift für die Aegyptischen Sprache, 61 (1926), p. 84-93

Borghouts, Joris F., The Magical Texts of the Papyrus Leiden I 348, Leiden, Brill, 1971.

—, Ancient Egyptian Magical Texts, Leiden, Brill, 1978 (Nisaba 9).

Brunner-Traut, Emma, "Tanz", in Lexicon der Aegyptologie, VI, hrsg. von W. Helck, E. Otto, Wiesbaden, Harrassowitz, 1986, col. 220-221.

—, Altägyptische Märchen, Köln, Diederichs, 1986.

-, Die Alten Aegypter, Verborgenes Leben unter Pharaoh, Stuttgart/ Berlin/Köln/Mainz, Kohlhammer, 19874.

Cauville, Sylvie, "Les statues cultuelles de Dendéra d'après les inscriptions pariétales", Bulletin de l'IFAO, 87 (1987), p. 73-117.

-, Dendera, III. Traduction, Leuven, Peeters, 2000 (Orientalia Lovaniensia Analecta 95).

-, Dendera, IV. Traduction, Leuven, Peeters, 2001 (Orientalia Lovaniensia Analecta 101).

-, Dendera, V-VI. Traduction. Les cryptes du temple d'Hathor, I, Leuven, Peeters, 2004 (Orientalia Lovaniensia Analecta 131).

Chappaz, Jean-Luc, "Quelques réflexions sur les conteurs dans la littérature égyptienne ancienne», in Hommages à François Daumas, 
Institut d'égyptologie de l'Université Paul Valéry, Montpellier, 1986, p. 104-108.

Chassinat, Emile, "A propos de deux tableaux du mammisi d'Edfou», Bulletin de l'IFAO, 10 (1912), p. 183-193.

—, Le temple de Deendera, III, Le Caire, IFAO, 1935

—, Le mammisi d'Edfou, Le Caire, IFAO, 1939.

Chassinat, Emile, Daumas, François, Le temple de Dendera, VII, Le Caire, IFAO, 1972.

Daumas, François, "Sur deux chants liturgiques des mammisis de

Dendera", Revue d'Egyptologie, 8 (1951), p. 31-46.

—, Les mammisis des temples égyptiens, Paris, Les Belles-Lettres, 1958.

—, Les mammisis de Dendera, Le Caire, IFAO, 1959.

—, "Les propylées du temple d'Hathor à Philae», Zeitschrift für Aegyptische Sprache, 95 (1968), p. 4-5.

Derchain-Urtel, Maria, Synkretismus in ägyptischer Ikonographie. Die Göttin Tjenenet, Wiesbaden, Harrassowitz, 1979 (Göttinger Orientforschungen IV, 8).

Desroches-Noblecourt, Christiane, «Un lac de turquoise, godets à onguents et destinées d'outre-tombe dans l'Egypte ancienne", Monuments Piots, 47 (1953), p. 1-34.

El SAady, Hassan, "Reflections on the Goddess Tayet", Journal of Egyptian Archeology, 80 (1994), p. 213- 217.

EL-SAYED, Ramadan, «Les rôles attribués à la déesse Neith dans certains Textes des Cercueils», Orientalia, 43 (1974), p. 275-294.

-, "Les sept vaches célestes, leur taureau et les quatre gouvernails", Mitteilungen des Deutsches Archäologisches Instituts Kairo, 36 (1980), p. 357-390.

Erman, Adolf, Grapow, Hermann, Wörterbuch der Aegyptischen Sprache, IV, Berlin, Akademie Verlag, 1982.

Frankfurter, David T. M., "Tabitha and the Apocalypse of Elijah", Journal of Theological Studies, 41 (1990), p. 13-25.

GNirs, Andrea, "Nilpferdstosszähne und Schlangenstäbe. Zu den magischen Geräten des so-genannten Ramesseumfundes", in Texte - Theben - Tonfragmente. Festschrift für G. Burkard, Wiesbaden, 2009, p. 128-155 (Aegypten und Altes Testament 76).

Goyon, Jean-Claude, Rituels funéraires de l'Ancienne Egypte, Paris, Le Cerf, 1997. 
Hari, Robert, «La grande-en-magie de la stèle du temple de Ptah à Karnak", Journal of Egyptian Archeology, 62 (1976), p. 100-107.

Helck, Wolfgang, "Die Erzählung vom verwunschenen Prinzen", in Form und Mass. Beiträge zur Literatur, Sprache und Kunst im Alten Aegypten. Festschrift G. Fecht, hrsg. von J. Osing, G. Dreyer, Wiesbaden, 1987, p. 218-225 (Aegypten und Altes Testament 12). Hodel-Hoenes, Sigrid, Leben und Tod im Alten Aegypten, Thebanische Privatgräber des Neuen Reiches, Darmstadt, Wissenschaftliche Buchgesellschaft, 1991.

Hornung, Erik, Geist der Pharaonenzeit, Zürich/München, Artemis Verlag, 1990.

Hubä̈, Peter, "Eine literarische Quelle der ägyptischer Religionsphilosophie. Das Märchen vom Prinzen der drei Gefahren zu überstehen hatte", in The Intellectual Heritage of Egypt. Studies Kakosy, ed. by U. Luft, Budapest, 1992, p. 277-300 (Studia Aegyptiaca 14).

Lalouette, Claire, Contes et récits de l'Egypte ancienne, Paris, Flammarion, 1995.

Lefebvre, Georges, Romans et contes égyptiens de l'époque pharaonique, Paris, rééd. Maisonneuve, 2003.

Leitz, Christian, Lexicon der Aegyptischen Götter und Götterbezeichnungen, Leuven/Paris, Dudley, Peeters, 2002 (Orientalia Lovaniensia Analecta 112).

-, Lexicon der Aegyptischen Götter und Götterbezeichnungen, V, Leuven/ Paris, Dudley, Peteers, 2002 (Orientalia Lovaniensia Analecta 114).

Lexa, François, La magie dans l'Egypte antique de l'Ancien Empire à l'époque copte, II, Les textes magiques, Paris, Geuthner, 1925.

Lichtheim, Myriam, Ancient Egyptian Literature, I, Berkeley/Los Angeles/Londres, University of California Press, 1975.

Macy Roth, Anne, Roenrig, Catherine, "Magical Bricks and the Bricks of Birth", Journal of Egyptian Archaeology, 88 (2002), p. 122-139.

Mariette, Auguste, Denderah: description générale du grand temple de cette ville, II, Paris, Franck, 1870.

-, Denderah: description générale du grand temple de cette ville, III, Paris, Franck, 1871. 
Maspero, Gaston, Les contes populaires de l'Egypte ancienne, Paris, Maisonneuve, 1882.

Miosi, Frank T., "God, Fate and Free Will in Egyptian Wisdom Literature", in Studies in Philology in honor of R. J. Williams, ed. by G. F. Kadish, G. E. Freeman, Toronto, SSEA Publications, 1982, p. 69-111.

Morenz, Siegfried, Untersuchungen zur Rolle des Schicksals in der ägyptischen Religion, Berlin, Akademie Verlag, 1960.

Papaikonomou, Irini, Huysecom-Haxhi, Stéphanie «Du placenta aux figues sèches: mobilier funéraire et votif à Thasos ", Kernos, 22 (2009), p. 133-158.

Pleyte, Willem, Rossi, Francesco, Papyrus de Turin, Leiden, Brill, 1869-1876.

Poo, Mu-Chou, Wine and Wine Offering in the Religion of Ancient Egypt, Londres/New York, Paul Kegan, 1995.

Posener, Georges, "Sur l'attribution du nom à un enfant", Revue d'Egyptologie, 22 (1970), p. 204-205.

Raue, Dietrich, "Die Sieben Hathoren von Prt», Annales du Service des Antiquités Egyptiennes, suppl. 34.2 (2005), p. 247-261.

Rochrolz, Matthias, Schöpfung, Feindvernichtung und Regeneration. Untersuchungen zum Symbolgehalt des machtgeladenes Zahl 7 im Alten Aegypten, Wiesbaden, Harrassowitz, 2002 (Aegypten und Altes Testament 56).

Sainte-Fare-Garnot, Jean, "Défis au destin», Bulletin de l'IFAO, 59 (1960), p. 1-28.

Sauneron, Serge, Le temple d'Esna, III, Le Caire, IFAO, 1968.

—, Les Prêtres de l'ancienne Egypte, Paris, Seuil, 1998.

Schmidt, Heike, Willeitner, Joachim, Nefertari Gemahlin Ramses'II, Mainz, Philip von Zabern, 1994.

Sснотт, Siegfried, Altägyptische Liebeslieder, Zürich, Artemis, 1950.

Schulman, Alan R., "A Birth Scene from Memphis", Journal of the American Research Center in Egypt, XXII (1985), p. 97-103.

Seeber, Christine, Untersuchungen zur Darstellung des Totengerichts im alten Aegypten, Münich, 1976 (Münchner Aegyptologische Studien 35).

Simon, Henrick, «Die Jungfrau im Turm. Zum historische Gehalt eines vermeintlichen Märchenmotivs in der Erzählung vom verwunschenen Prinzen", in Texte - Theben - Tonfragmente. Festschrift 
für G. Burkard, Wiesbaden, Harrassowitz, 2009, p. 385-398 (Aegypten und Altes Testament 76).

Spieser, Cathie, "Le sang et la vie éternelle dans le culte solaire amarnien", in Actes du $9^{e}$ Congrès International des Egyptologues, éd. par Ch. Cardin, J.-C. Goyon, Leuven, Peteers, 2007, p. 17191728 (Orientalia Lovaniensia Analecta 150).

Staehelin, Elisabeth, "Bindung und Entbindung. Erwägungen zu Pap. Westcar 10.2», Zeitschrift für Ägyptische Sprache, 96 (1970), p. 125-139.

—, "Menit», in Lexicon der Ägyptologie, IV, hrsg. von W. Helck, E. Otto, Wiesbaden, Harrassowitz, 1982, col. 52-53.

Vernus, Pascal, "Namensgebung", Lexicon der Aegyptologie, IV, hrsg. von W. Helck, E. Otto, Wiesbaden, Harrassowitz, 1982, col. 326-333.

Wegner, Josef, "A Decorated Birth-brick from South Abydos", Egyptian Archaeology, 21 (2002), p. 3-4.

Yamazaki, Naoko, Zaubersprüche für Mutter und Kind: Papyrus Berlin 3027, Berlin, Achet Verlag, 2003.

\section{Crédits iconographiques}

Fig. 1:

D'après Brunner-Traut, Emma, Die Alten Aegypter, Verborgenes Leben unter Pharaoh, Stuttgart/Berlin/Köln/Mainz, Kohlhammer, 19874, p. 58 , fig. 13 .

Fig. 2:

D'après Dondelinger, Emile, Le Livre sacré de l'Ancienne Egypte. Papyrus d'Ani BM 10470, éd. par Ph. Lebaud, Vesoul, 1987, p. 28 (détail).

Fig. 3-4:

D’après Mariette, Auguste, Denderah: description générale du grand temple de cette ville, II, Paris, Franck, 1870, pl. 43b et 59. 
Fig. 5:

D'après Chassinat, Emile, Daumas, François, Le temple de Dendera, III, Le Caire, IFAO, 1972, pl. CLXXVI.

Fig. 6:

D’après Daumas, François, Les mammisis de Dendera, Le Caire, IFAO, 1959, pl. XLIII.

Fig. 7:

D’après Chassinat, Emile, "A propos de deux tableaux du mammisi d'Edfou", Bulletin de l'IFAO, 10 (1912), pl. XXXII. 